\title{
Urban Dispersion Modelling Capabilities Related to the UDINEE Intensive Operating Period 4
}

\author{
Piotr Kopka ${ }^{1}$ D $\cdot$ Slawomir Potempski ${ }^{1}$ Aleksej Kaszko ${ }^{2} \cdot$ Michal Korycki $^{1}$
}

Received: 20 February 2018 / Accepted: 9 October 2018 / Published online: 7 November 2018

(c) The Author(s) 2018

\begin{abstract}
The Quick Urban and Industrial Complex (QUIC) atmospheric transport and dispersion modelling system, developed by the Los Alamos National Laboratory, is evaluated using measurement data from the Joint Urban 2003 gas-tracer measurements conducted in Oklahoma City, USA. This activity has been coordinated within the Urban Dispersion International Evaluation Exercise (UDINEE) project, led by the European Commission-Joint Research Centre. Four different set-ups for the QUIC program are evaluated using different types of wind-speed data, such as local onsite measurements and flow fields produced by the Weather Research and Forecasting mesoscale model. The simulation results are evaluated against measured data for instantaneous puff releases from intensive operation period 4 of the Joint Urban 2003 field experiment. The selection of performance measures is based on the assumptions made for the UDINEE project. The differences in the results of simulations for various set-ups are described.
\end{abstract}

Keywords Industrial complex · Joint Urban 2003 experiment · Quick Urban and Industrial complex · Weather Research and Forecasting model

\section{Introduction}

The release of chemical or radiological substances in an urban environment can lead to significant consequences, and may have huge social, economic and health impacts. Therefore, the proper prediction of the dispersion of harmful material released into the atmosphere is an

Piotr Kopka

piotr.kopka@ncbj.gov.pl

Slawomir Potempski

slawomir.potempski@ncbj.gov.pl

Aleksej Kaszko

aleksej.kaszko@ncbj.gov.pl

1 National Centre for Nuclear Research, Division of Nuclear Energy and Environmental Studies, Otwock, Poland

2 Institute of Heat engineering, Warsaw University of Technology, Warsaw, Poland 
important task. However, due to the complexity of urban areas, such a task cannot be realized by applying relatively simple dispersion models.

The presented study and analysis were realized within the Urban Dispersion International Evaluation Exercise (UDINEE) project, led by the Joint Research Centre (JRC European Commission) (Hernández-Ceballos et al. 2017). The main goal of the project is to evaluate various urban atmospheric dispersion models for use in emergency preparedness and response. Each of the models has its own advantages and disadvantages, and the comparison is essential for understanding their behaviour. To validate the models presented by different teams, the organizers provided data from the Joint Urban 2003 (JU2003) field experiment held in Oklahoma City, USA (Clawson et al. 2005). During the JU2003 experiment, personnel from the National Oceanic and Atmospheric Administration Air Resources Laboratory Field Research Division conducted multiple instantaneous puff releases of sulphur hexafluoride gas $\left(\mathrm{SF}_{6}\right)$ in the downtown area of Oklahoma City. Each release occurred at a different time of the day and the weather conditions varied throughout the experiment.

The typical modelling of dispersion in an urban environment consists of two main components. The first part includes wind-field simulations describing the meteorological situation within the domain. The models are used to simulate the contaminant dispersion with application of meteorological fields, while a number of simulations can be performed using different models, modelling options and data initialization. In many cases, the wind field is estimated using empirical models defined by the specification of the vertical profiles (Röckle 1990) or by models applying advanced computational fluid dynamics (CFD) (Brown et al. 2013). The dispersion component can be performed using either a Lagrangian particle dispersion model (Wilson et al. 2009) or a Eulerian dispersion model (Wyszogrodzki et al. 2012).

Our aim is to describe the results of dispersion simulations performed with the Quick Urban and Industrial Complex (QUIC) model for the UDINEE project, which is a tool developed by the Los Alamos National Laboratory to perform rapid computations of transport and dispersion of gas and/or particle releases near buildings. The theoretical basis of the QUIC model and a User Guide can be found in Pardyjak and Brown (2003) and Williams et al. (2004). To validate the QUIC calculations, different set-ups have been used combining the QUIC-URB, QUIC-PLUME or QUIC-CFD models together with meteorological data gathered from the Portable Weather Information Data System (PWIDS) sensor 15 operated during the JU2003 experiment, or from results from the Weather Research and Forecasting (WRF) model. Each proposed set-up contains various components of the flow and gastransport models. In addition, various meteorological input data have been used. The results of simulations have been verified against measured concentrations at the locations of sensors operated during the JU2003 experiment.

The QUIC-URB model is a fast-response model usually used for rapid computation of three-dimensional flow fields around building complexes, using its own empirical algorithms and mass-conservation scheme (see Röckle 1990). The QUIC-PLUME model is adapted for use in the inhomogeneous environments of cities, while the Lagrangian random-walk dispersion model is used for computing concentration and deposition fields around buildings (see Williams et al. 2004). The QUIC-CFD model is one of the flow solvers implemented by the Los Alamos National Laboratory in the QUIC program, and is a simple CFD code for solving the Navier-Stokes equations by applying the pressure Poisson equation. The QUIC-CFD model uses a single-equation turbulence model (Gowardhan et al. 2011) with a diagnostic turbulent length scale and the artificial compressibility technique of Chorin (1968).

Nelson et al. (2016) performed a similar study focusing on the flow fields, in which they evaluated the QUIC model against the JU2003 tracer-gas measurements with the use of simulated wind fields by the WRF model, and local wind-speed measurements (from 
the PWIDS-15 sensor). Nelson et al. (2016) evaluated puff releases from different intensive operation periods (IOP) than those presented in Sect. 2. However, one of their findings was that building effects have a strong influence over the plume spread. We analyze different puff releases and model settings, concentrating on the dispersion results. There are several key differences between our study and that of Nelson et al. (2016), including model set-up details, such as the number of tracer particles, the domain size, the grid resolution, and the choice of flow model. Therefore, the following analysis and that of Nelson et al. (2016) can be considered as complementary.

In Sect. 2, we describe the JU2003 experiment and measurements used for model comparison. In Sect. 3, we briefly describe the QUIC program, with details on the evaluation of the WRF model, the meteorological measurements, the computational domain setting, and the specification of the models and data used in the analysis. In Sect. 4, we define the measures used for model-performance evaluation, and the results of all dispersion simulations, including the analysis of the individual puffs and the prediction-observation relationships, are presented and discussed in Sect. 5. A summary and conclusions are given in Sect. 6.

\section{Description of the Joint Urban 2003 Experiment, Intensive Operating Period 4}

The JU2003 field experiment was conducted in Oklahoma City from 28 June to the end of July 2003, and was sponsored by the U.S. Defense Threat Reduction Agency (DTRA) (see Clawson et al. 2005), with the central goal the collection of measurements necessary for the evaluation of urban transport and dispersion models. During 10 IOPs in downtown Oklahoma City, usually four instantaneous puffs of sulfur hexafluoride $\mathrm{SF}_{6}$ gas were released by bursting 2 -m diameter balloons during the day or night. Concentration measurements were taken over a 1-h period, beginning at the release time, and extending $20 \mathrm{~min}$ beyond the third release.

The three IOP-4 releases were conducted near the Botanical Gardens in downtown Oklahoma City as indicated by the green mark in Fig. 1. The initial release of IOP 4 (hereafter termed puff 4.1) occurred at 1400 UTC on 9 July 2003, and the mass of released substance during this puff was $0.996 \mathrm{~kg}$. The second release (puff 4.2) began $20 \mathrm{~min}$ later, and the mass of the released substance was $1.002 \mathrm{~kg}$. The releases ended with the last puff (puff 4.3) conducted at 1440 UTC with a mass of $0.504 \mathrm{~kg}$ of released substance. The locations of the source and sensors are presented in Fig. 1. The model testing here is based on the set of measured and simulated $\mathrm{SF}_{6}$ concentration time series with a temporal resolution of $0.5 \mathrm{~s}$. The sensor locations for each of the puffs are denoted as follows: Puff 4.1 sensors are L01, L08, L13 and L15; Puff 4.2 sensors are L01, L08, L11, L13 and L17; Puff 4.3 sensors are L01 and L11. This intensive observation period is analyzed because of the relatively large amount of good quality measurements and its lack of attention so far in the literature.

\section{The Quick Urban and Industrial Complex Model}

The QUIC Dispersion Modeling System has been developed by the Los Alamos National Laboratory to perform quick computations of the transport and dispersion of gas and/or particle releases in areas near buildings (Pardyjak and Brown 2003; Williams et al. 2004). The program consists of an empirical-diagnostic wind solver (QUIC-URB), a Lagrangian random-walk model (QUIC-PLUME) adapted to urban areas, and a graphical user interface 


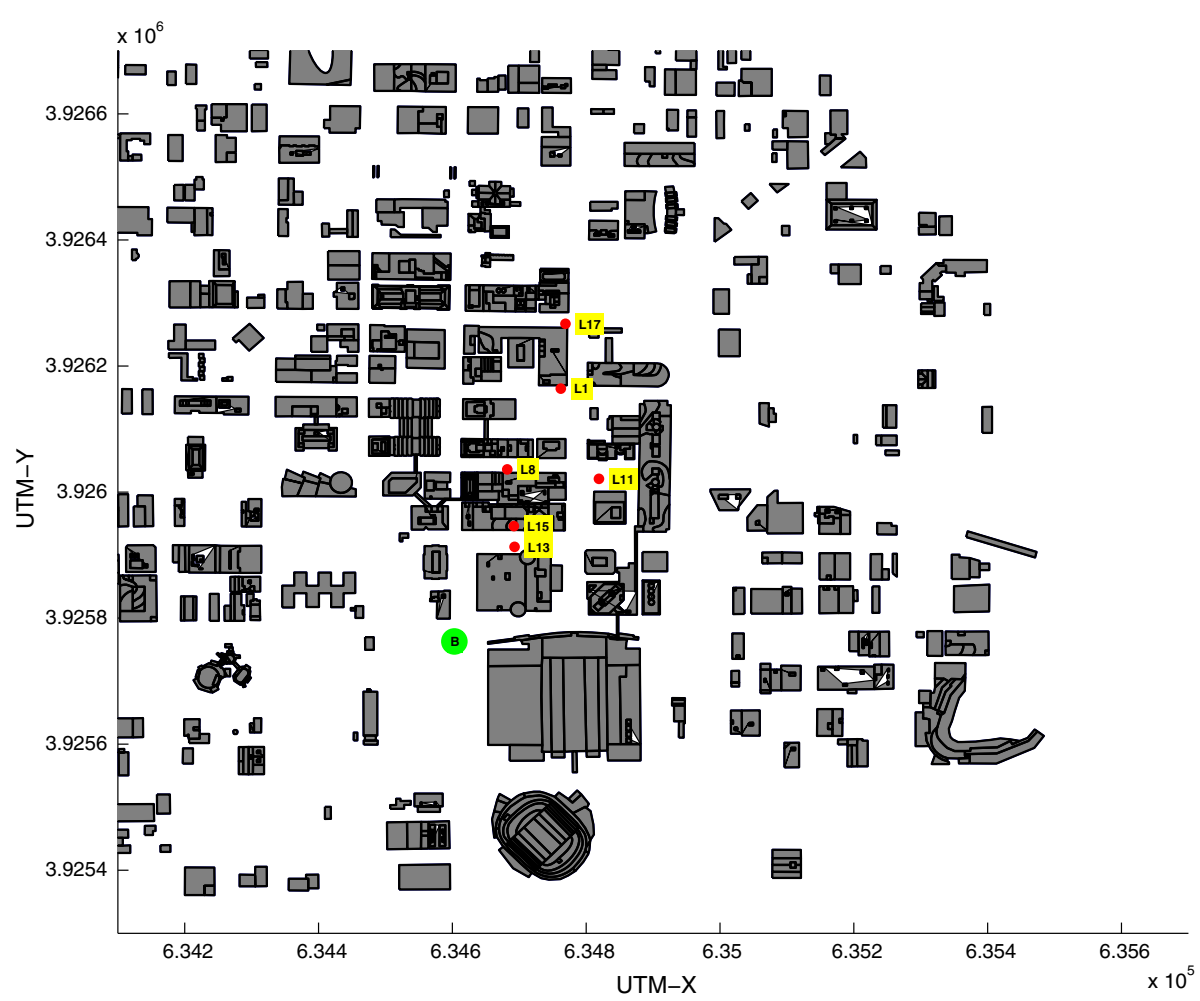

Fig. 1 Map of the locations of the gas-tracer samplers deployed in Oklahoma City during the Joint Urban 2003 experiment IOP 4 . Sampler sites are indicated by red marks denoted with $L$ - labels, and the release location is indicated by the letter B (botanical) within a green circle

(QUIC-GUI). The aim of the QUIC-URB interface is to perform quick simulations of the three-dimensional flow fields around buildings, for which empirical algorithms and a massconservation model are employed. The QUIC-PLUME dispersion model is adapted for the non-uniform environment of cities. The QUIC-GUI program enables the user to easily define source parameters, create the geometry of buildings, and enter meteorological input data.

\subsection{Quick Urban and Industrial Complex Geometry Set-up}

The three-dimensional building data were provided by the UDINEE project coordinators. For the set-up of the geometry within the model, the Landscape_Building_v11_JEF file in .shp format was used. The average building height in the Oklahoma City downtown area (the central business district) is about $27 \mathrm{~m}$, and there are several skyscrapers located in this area. The tallest buildings are the Bank One Building (152 m), the FNC Building (123 m), the Oklahoma Tower (117 m), the Kerr-McGee Building (115 m), and City Place $(107 \mathrm{~m})$. The data were added to the QUIC program with the "automatic city generation from building shape file" tool. The domain input data consist of the domain size $1600 \mathrm{~m} \times$ $1400 \mathrm{~m} \times 402 \mathrm{~m}(X \times Y \times Z)$ covering most of the central business district of Oklahoma City, and a grid size of $1 \mathrm{~m} \times 1 \mathrm{~m} \times 1 \mathrm{~m}(d x \times d y \times d z)$. The spatial orientation axes have been defined as follows: $X$ (west-east) and $Y$ (south-north). Georeference data were needed to 


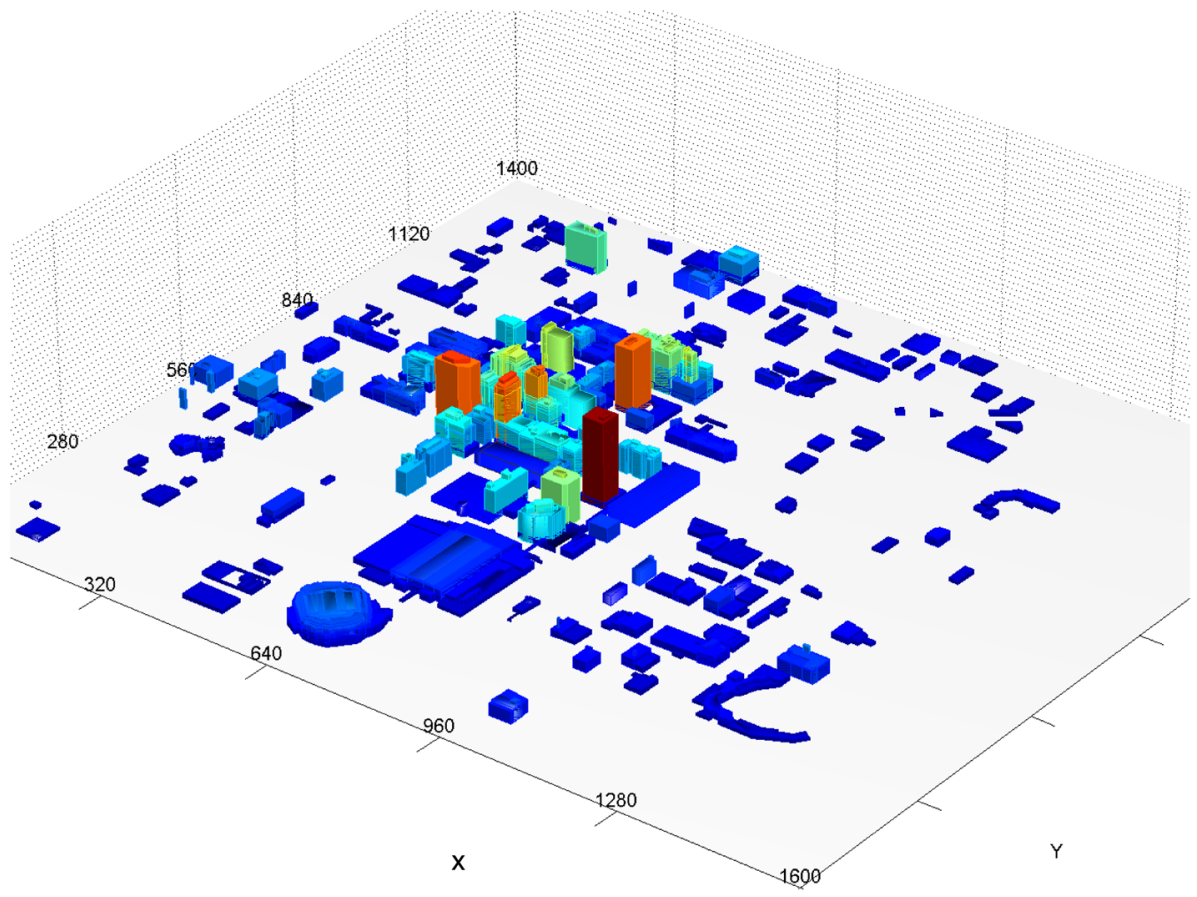

Fig. 2 Three-dimensional view of the domain used in the QUIC simulations. The different colours of buildings represent their maximum height, ranging from dark blue (small buildings) to dark red (tall buildings)

provide the coordinates of the origin in the Universal Transverse Mercator system, with the following coordinates: $U T M X-634.1 \mathrm{~m}, U T M Y-3925.3 \mathrm{~km}$; and Universal Transverse Mercator Zone 14N. After generating the three-dimensional geometry (see Fig. 2), several modifications were made, namely: 1) adding the building connectors presented in Fig. 3a and $b$, because connectors can have a strong influence on the flow; 2) changing the type of one of the buildings to a parking garage, as one of the sensors was located there (see Fig. 3c and d).

\subsection{The QUIC-URB Flow Solver}

The QUIC-URB flow solver is an empirically-based diagnostic flow model (Röckle 1990). The model domain is first initialized without buildings by creating either a horizontally homogeneous wind field using an inflow profile, or a horizontally inhomogeneous wind field from multiple wind profiles at different locations within the domain by applying inversesquare-law interpolation and mass consistency constraints. The domain is then overlaid with buildings, and the size and shape of flow regions developing around the buildings, as well as the initial flow within the zones (e.g., upwind rotor, downwind cavity and wake, street-canyon vortex, and rooftop vortex), are computed by applying various empirical relationships based on the building height, width, and length, as well as the spacing between buildings. This initial flow field is then forced to satisfy mass-consistency constraints following Sherman (1978). More information about the QUIC-URB flow solver can be found in Pardyjak and Brown (2003). 
(a)

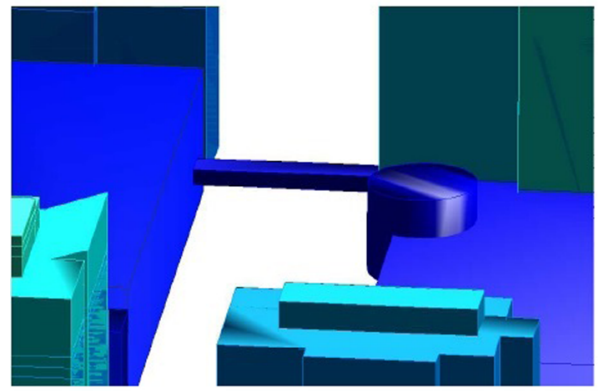

(c)

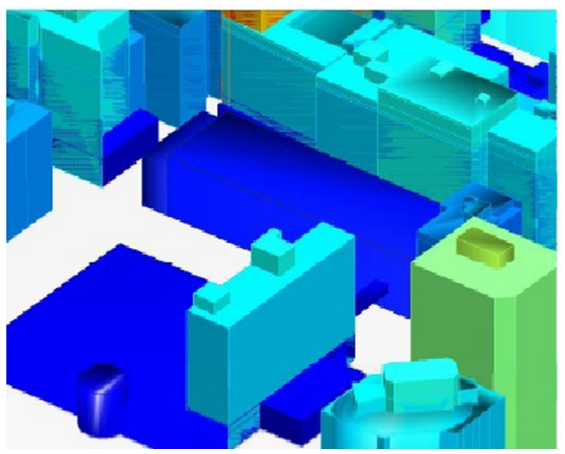

(b)

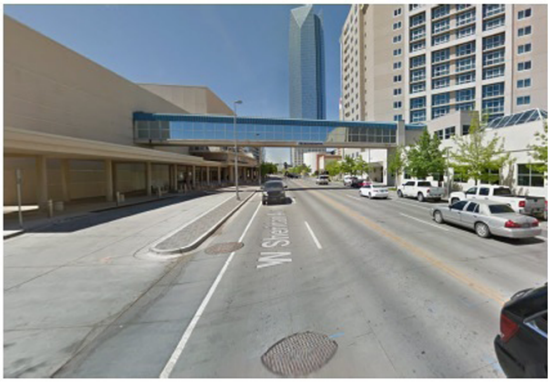

(d)

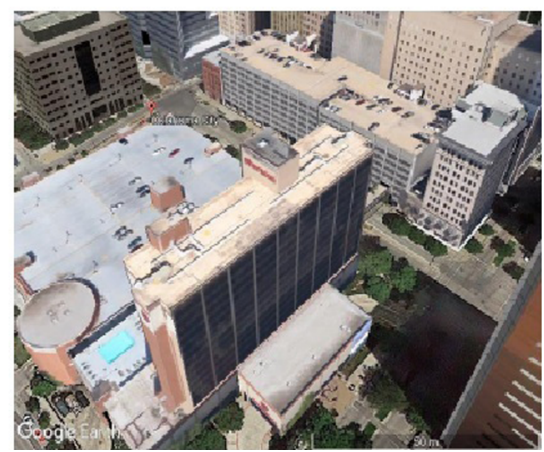

Fig. 3 a Three-dimensional view of the building connector used in the QUIC simulations; $\mathbf{b}$ Google Maps view of the same building connector; $\mathbf{c}$ three-dimensional view of the parking garage used in the QUIC simulations; d Google Map views of the parking garage

\subsection{IOP 4 QUIC-URB Meteorological Input Set-up}

During the JU2003 experiment, meteorological quantities were measured continuously at nearly 100 locations near and around the Oklahoma City downtown area. Wind-speedmeasurement devices in this experiment were situated in most cases at heights $1-1.5 \mathrm{~m}$, and approximately 20 anemometers were placed on top of buildings in the central business district at heights ranging from 12-148 $\mathrm{m}$. We use meteorological data obtained from the post office station PWIDS-15 located $1 \mathrm{~km}$ upwind to the centre of domain at a height of 40 $\mathrm{m}$. This sensor was selected because of its height and the wind speeds are not disturbed by barriers, such as tall buildings. Wind speeds and directions for the puff releases during IOP 4 are summarized in Table 1, noting that in the simulations, 1-min averaged data were used.

\subsection{Mesoscale Model Set-up}

The WRF model version 3.8.1 was configured for five domains as shown in Fig. 4, illustrating the mother domain and four nested domains. The white box in Fig. 4 represents the first nested domain with a $27-\mathrm{km}$ resolution, the red box shows the 9-km resolution domain, green the $3-\mathrm{km}$ resolution domain, and the last domain, shown in blue, has the finest resolution of 1 $\mathrm{km}$, whose results were used to drive the simulations of the QUIC-URB model. As the first puff of IOP 4 was released at 1400 UTC on 9 June, calculations were started at 0000 UTC 
Table 1 Wind speed and direction from the PWIDS-15 station used for simulations

\begin{tabular}{|c|c|c|c|c|c|c|}
\hline \multirow[t]{2}{*}{ Time } & \multicolumn{2}{|l|}{ Puff 4.11400} & \multicolumn{2}{|l|}{ Puff 4.21420} & \multicolumn{2}{|l|}{ Puff 4.31440} \\
\hline & Speed $\left(\mathrm{m} \mathrm{s}^{-1}\right)$ & Direction & Speed $\left(\mathrm{m} \mathrm{s}^{-1}\right)$ & Direction & Speed $\left(\mathrm{m} \mathrm{s}^{-1}\right)$ & Direction \\
\hline+0 & 8.3 & 222 & 6.8 & 203 & 4.9 & 208 \\
\hline+1 & 5.0 & 213 & 5.1 & 217 & 5.5 & 212 \\
\hline+2 & 3.9 & 208 & 6.2 & 205 & 7.0 & 223 \\
\hline+3 & 6.0 & 196 & 6.5 & 207 & 6.7 & 214 \\
\hline+4 & 8.5 & 205 & 6.3 & 212 & 4.9 & 209 \\
\hline+5 & 7.4 & 210 & 6.9 & 201 & 8.2 & 204 \\
\hline+6 & 6.4 & 211 & 6.9 & 203 & 8.0 & 204 \\
\hline+7 & 7.2 & 216 & 6.4 & 199 & 7.6 & 209 \\
\hline+8 & 8.3 & 215 & 7.0 & 207 & 6.8 & 205 \\
\hline+9 & 7.8 & 212 & 5.1 & 206 & 7.6 & 208 \\
\hline+10 & 8.0 & 209 & 5.0 & 205 & 6.8 & 202 \\
\hline+11 & 5.3 & 208 & 6.3 & 209 & 6.1 & 204 \\
\hline+12 & 6.2 & 217 & 5.4 & 224 & 6.3 & 194 \\
\hline+13 & 6.6 & 219 & 6.3 & 231 & 6.6 & 201 \\
\hline+14 & 7.1 & 206 & 4.4 & 215 & 6.4 & 202 \\
\hline+15 & 6.6 & 202 & 4.9 & 208 & 6.5 & 198 \\
\hline+16 & 7.9 & 201 & 6.5 & 213 & 5.2 & 200 \\
\hline+17 & 7.1 & 216 & 7.3 & 194 & 4.9 & 193 \\
\hline+18 & 7.5 & 196 & 8.0 & 198 & 5.4 & 208 \\
\hline+19 & 7.0 & 198 & 5.9 & 205 & 7.3 & 195 \\
\hline+20 & 6.8 & 203 & 4.9 & 208 & 7.8 & 198 \\
\hline
\end{tabular}

The time column indicates how often the wind and turbulence fields were updated during the 20-min simulation. The next columns indicate the wind speed and direction in the time interval between 1400 and 1500 . Wind speed and direction data from PWIDS-15 were averaged over 1-min time intervals. The frequency of the original data is $10 \mathrm{~s}$

to reduce the effects of a cold-start and to capture all puffs in one mesoscale simulation, giving a longer simulation time than in Wyszogrodzki et al. (2012). The JU2003 experiment has been successfully simulated with a combination of WRF and Eulerian/semi-Lagrangian computational flow models. Here, a total of $24 \mathrm{~h}$ of simulation was conducted with the nonhydrostatic option in every domain using the National Centers for Environmental Prediction Global Forecasting System input data of 81-km resolution. Every domain has 40 eta-levels reaching $100 \mathrm{hPa}$ at the upper boundary. The last domain was set to include Oklahoma City in the middle (blue box in Fig. 4). Every nested domain has three times higher resolution than its parent domain.

\subsection{Weather Research and Forecasting Model Results}

While the resolution of the finest domain of the WRF model was set to $1 \mathrm{~km}$, performing a simulation at such a scale using a mesoscale model is not a straightforward task, as it requires high-resolution geospatial data, which are not widely available. Moreover, when the mesoscale model results are used as boundary conditions for a city-scale model, mul- 


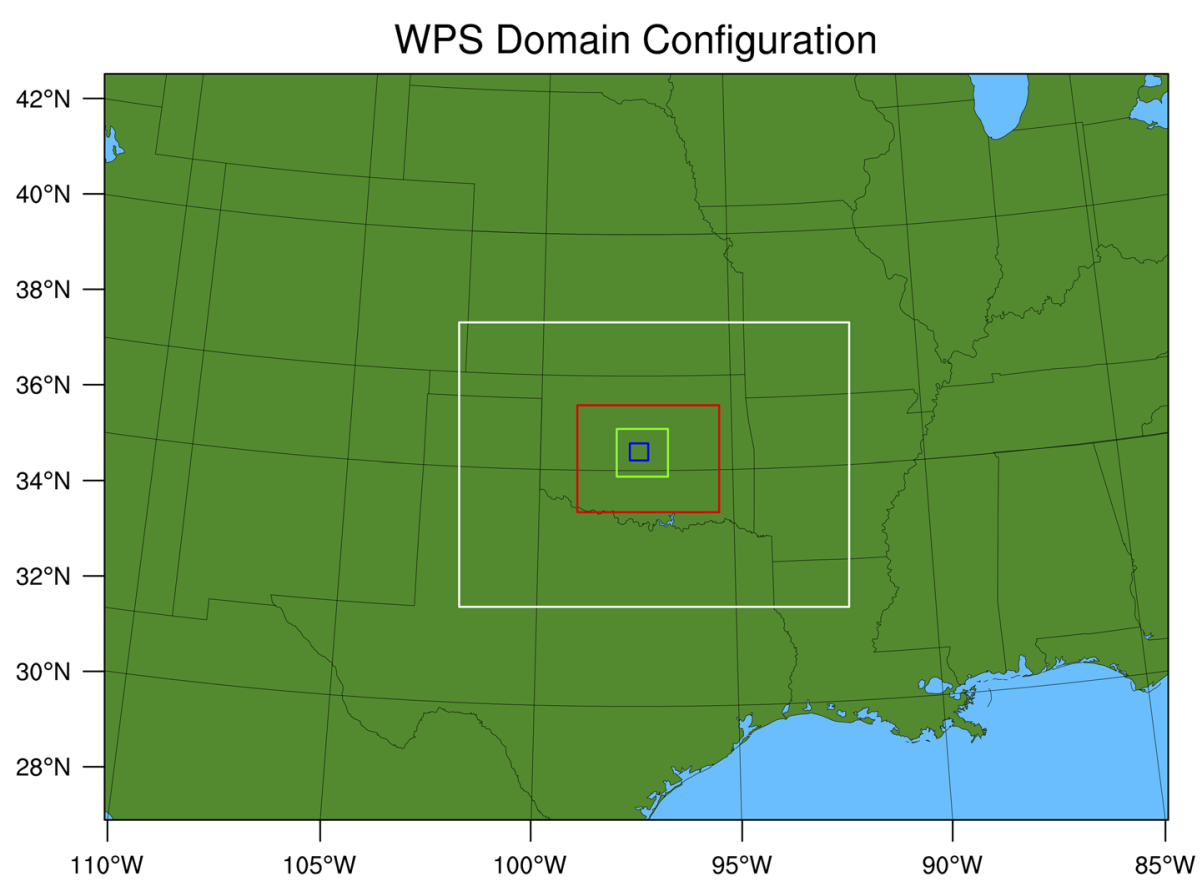

Fig. 4 The WRF model computational domain generated by the WRF preprocessing system. The whole area of the figure is domain 1 . The white box shows domain 2 , the red is domain 3, the light green is domain 4, and dark blue is the domain 5 of spatial resolutions $81 \mathrm{~km}, 27 \mathrm{~km}, 9 \mathrm{~km}, 3 \mathrm{~km}$, and $1 \mathrm{~km}$, respectively

tiscale problems such as inter-scale feedbacks arise. We have performed simulations using only widely available geospatial and meteorological data to investigate whether coupling a mesoscale model with the QUIC model is a good starting point for further research, such as for operational use in emergency-response problems. Figure 5 presents the WRF model results from the finest domain compared with the PWIDS-15 anemometer from 1400 UTC to 1500 UTC, corresponding to the duration of the QUIC dispersion simulation for that period. Figure 5 shows that the PWIDS-15 anemometer measurements are strongly influenced by smaller scales, as the time series are much more complicated than the WRF model results. However, the WRF model seems to follow the overall trend of both the wind speed and direction time series. The biggest discrepancy between the WRF model and PWIDS-15 measurements is found in the last $10 \mathrm{~min}$ of the wind-direction signal. However, approximately $10^{\circ}$ of wind-direction discrepancy should not cause large differences in dispersion. According to Hanna et al. (2006), small differences in the incoming wind direction may cause minor changes in the flow within the urban canopy and the dispersion.

\subsection{QUIC System Test Cases}

Described here are the four different set-ups of the QUIC model.

\section{- Case Q1 (the QUIC-PWIDS-URB-PLUME set-up)}

Case Q1 uses as input the data from the sensor PWIDS-15 shown in Table 1. The QUIC program enables various physics options and the choice of some algorithms. In the QUIC-URB system, the inner-grid surface roughness was set to $0.1 \mathrm{~m}$, the convergence 

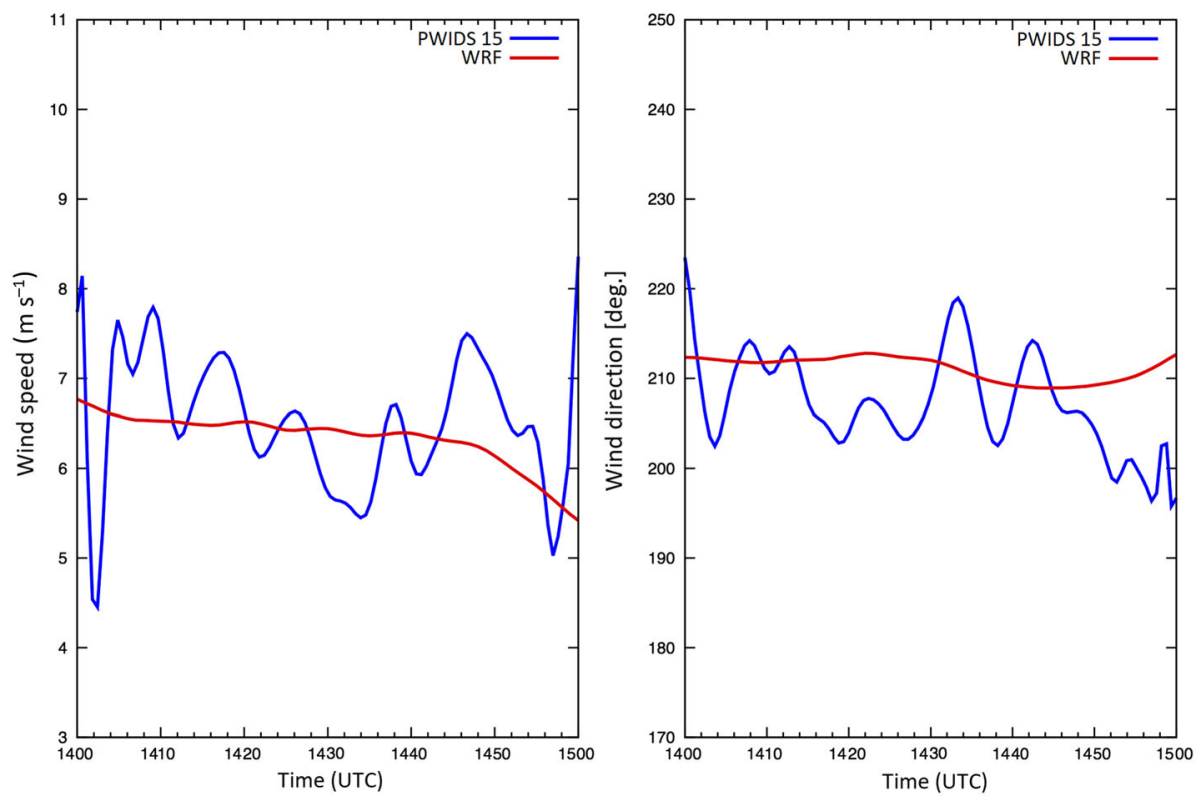

Fig. 5 Wind speed and direction from the WRF model (red) in comparison with smoothed PWIDS 15 anemometer measurements (blue)

criterion was set to a maximum of 500 iterations, while the residual reduction in the order of magnitude was set to three, which is recommended in Pardyjak and Brown (2003). The following algorithms recommended by QUIC developers were also used. The Rockle with Fackrell cavity-street-canyon algorithm, the area-scaled wake algorithm, the blended-area algorithm, the recirculation-rooftop algorithm, and the highrise-modifedvortex parametrization for the upwind-cavity algorithm.

The street-canyon algorithm is applied where a building is found within the cavity zone of another building, with the cavity length used to determine where this algorithm is applied. The blended-region algorithm, also known as the intersection algorithm, is used to interpolate the flow around street canyons. The modified-highrise-vortex parametrization inserts a vortex and retardation region with a limitation of the vortex size for high buildings. For more details on the methods used, see Pardyjak and Brown (2003).

The QUIC-PLUME parameters are identical for all simulations. The collecting box resolution in the $x, y$, and $z$ directions was set to $5 \mathrm{~m} \mathrm{cell}^{-1}, 5 \mathrm{~m} \mathrm{cell}^{-1}$, and $\approx 2 \mathrm{~m}$ cell $^{-1}$, respectively. The $z$ resolution was set to $2 \mathrm{~m} \mathrm{cell}^{-1}$ as the upper limit on the $z$ axis was $402 \mathrm{~m}$. We use the maximum number of particles able to be applied in simulations, taking into account some computational constraints, resulting in the use of $5 \times 10^{6}$ particles. The mass of $\mathrm{SF}_{6}$ distributed to each model particle is proportional to the total $\mathrm{SF}_{6}$ mass released.

The duration of each puff simulation was $20 \mathrm{~min}$, the timestep was $0.1 \mathrm{~s}$, and the concentration averaging time and particle output was set to $0.5 \mathrm{~s}$. Because the puffs were released and dispersed throughout the domain within a 1-h time window, the weather conditions, such as ambient temperature, ambient pressure, surface temperature, and ambient relative humidity are more or less constant. 


\section{- Case Q2 (QUIC-WRF-URB-PLUME)}

The Q2 case differs from the Q1 case only in the origin of the meteorological input data, for which the outputs from the WRF model presented in Sect. 3.4 are used. The proper meteorological generator is configured to use the WRF data for multi-sensor inputs. The whole domain in this case (shown in Fig. 4 as the blue domain) was divided into sectors, with twelve grid model nodes used in the QUIC-PLUME model acting as sensor inputs.

\section{- Case Q3 (QUIC-PWIDS-CFD-PLUME)}

The Q3 case is a CFD variant of the QUIC model instead of the URB model to produce the three-dimensional wind field used by the QUIC-PLUME model. The other parameters are the same as in the Q1 case. The QUIC-CFD model is generally faster than other commonly used CFD codes, but not as fast as the QUIC-URB model, which can handle complex geometries since it uses the governing equations instead of empirical parametrizations to reproduce the effects of buildings on the flow field. The QUIC program automatically generates values for the CFD parameters based on the grid cell size, mean wind speed, the size of the domain and the average building height. The parameter $d t(s)$ is the CFD model timestep set to satisfy the Courant condition. The convergence timestep is the number of steps required for the model to converge to a steady-state solution. The parameter Lmix is the maximum allowable turbulent mixing length, whose value is based on the average building height. The Press - Iter parameter value defines the number of pressure iterations performed for each timestep. For a simple simulation, which includes the domain with two buildings, three to five pressure iterations should be sufficient, but larger values may be required for very large simulation domains, such as for the JU2003 experiment. In our case, $d t=0.04$, giving 5,471 convergence timesteps, the maximum turbulent mixing length scale Lmix $=8.7 \mathrm{~m}$, and the pressure iteration was set to 15 , corresponding to the number of buildings in the computational domain.

\section{- Case Q4 (QUIC-PWIDS-CFD-CFD)}

The QUIC-CFD model gives the option of using the turbulence fields produced by the QUIC-CFD solver and/or using the default turbulence model inside of the QUICPLUME model. In the Q4 case, the QUIC-CFD model is used as the flow and turbulence model, with the input parameters set as for the Q3 case: $d t=0.04$, the convergence timestep $=5,471, \operatorname{Lmix}=8.7 \mathrm{~m}$, and pressure-iteration number was set to 15 .

\section{Measures of Performance}

For the analysis, we consider data from a network of real-time concentration samplers, and simulated values at the corresponding sensor locations. Measured and modelled data are denoted as $C_{o}$ (observations) and $C_{p}$ (predictions), respectively. The whole set of modelled data and observations in time for a single $L$ station have the form $C_{o} \equiv\left(C_{o}^{1}, C_{o}^{2}, \ldots, C_{o}^{N}\right)$ and $C_{p} \equiv\left(C_{p}^{1}, C_{p}^{2}, \ldots, C_{p}^{N}\right)$. The upper index identifies the timestep, $N$ indicates the total number of pairs, where $C_{o}^{i} \neq-9$ and $C_{p}^{i} \neq-9$. The -9 values correspond to corrupted samples, invalid or unspecified $C^{i}$ values. The overbar symbol $\bar{C}_{p}$ and $\bar{C}_{o}$ denotes the mean over the given dataset (for example, $\bar{C}_{o}=\frac{1}{N} \sum_{i=1}^{N} C_{o}^{i}$ ). The parameters $\sigma_{C_{p}}$ and $\sigma_{C_{o}}$ represent the standard deviation (i.e. $\sigma_{C_{o}}=\sqrt{\left.\frac{\sum_{i=1}^{N}\left(C_{o}^{i}-\bar{C}_{o}\right)^{2}}{N-1}\right)}$. 
The analysis incorporates several statistical measures of performance suggested by the UDINEE project (see Chang and Hanna 2005): the factor-of-two metric,

$$
F A C 2=\frac{N_{2}}{N}
$$

where $N_{2}$ is the number of pairs where the condition $0.5 \leq C_{p}^{i} / C_{o}^{i} \leq 2$ is fulfilled, and $N$ is the number of all timesteps for which measurements and predictions exist. The fractional bias is

$$
F B=2 \frac{\left(\bar{C}_{o}-\bar{C}_{p}\right)}{\left(\bar{C}_{o}+\bar{C}_{p}\right)},
$$

which can be expressed as the difference $F B=F B_{F N}-F B_{F P}$, where $F B_{F N}$ can be considered as the underpredicting (false-negative) component of $F B$, where $F B_{F P}$ can be considered as the overpredicting (false-positive) component of $F B$ corresponding to

$$
F B_{F N}=\frac{\frac{1}{2} \sum_{i=1}^{N}\left[\left|C_{o}^{i}-C_{p}^{i}\right|+\left(C_{o}^{i}-C_{p}^{i}\right)\right]}{\frac{1}{2} \sum_{i=1}^{N}\left(C_{o}^{i}+C_{p}^{i}\right)}
$$

and

$$
F B_{F P}=\frac{\frac{1}{2} \sum_{i=1}^{N}\left[\left|C_{o}^{i}-C_{p}^{i}\right|+\left(C_{p}^{i}-C_{o}^{i}\right)\right]}{\frac{1}{2} \sum_{i=1}^{N}\left(C_{o}^{i}+C_{p}^{i}\right)},
$$

respectively. The geometric mean bias is

$$
M G=\exp \left(\overline{\ln C_{o}}-\overline{\ln C_{p}}\right),
$$

the normalized mean square error is

$$
N M S E=\frac{\frac{1}{N} \sum_{i=1}^{N}\left(C_{o}^{i}-C_{p}^{i}\right)^{2}}{\left(\bar{C}_{o} \bar{C}_{p}\right)},
$$

and the correlation coefficient $(R)$ is defined as the ratio between the covariance and the standard deviation product,

$$
R=\frac{\frac{1}{N} \sum_{i=1}^{N}\left(C_{o}^{i}-\bar{C}_{o}\right)\left(C_{p}^{i}-\bar{C}_{p}\right)}{\left(\sigma_{C_{o}} \sigma_{C_{p}}\right)} .
$$

These metrics are used to evaluate and compare the performance of the models, with each metric focused on a separate aspect of the evaluation. In a perfect model, the values of $M G$ and $F A C 2=1$, while for the ideal values of $F B, M G$ and $N M S E=0$. Pearson's correlation coefficient can take values in the range between -1 (perfect negative linear correlation) and 1 (perfect positive linear correlation), while $R=0$ implies no correlation between the variables. For urban conditions, the typical magnitudes of the above performance measures and estimates of model acceptance criteria are as follows: FAC2 $>0.3,|F B|<0.67$ and $N M S E<6$ (Hanna and Chang 2012).

The calculation range is limited to timesteps at each sensor with a concentration over a given threshold value, as this enables an easier comparison of important parameters, such as the peak concentration. The concentration threshold filters out irrelevant values that would otherwise bias the statistical metrics. In our case, we took into account only the timesteps between when the measured and corresponding modelled concentration values first exceed the concentration threshold (with the threshold exceeded by either the 
measurement or the model prediction), and the first time where both measurement and model values fall below the concentration threshold, with these times described here as, respectively, $T_{0}$ and $T_{1}$ :

$$
\begin{aligned}
& T_{0}=\operatorname{minimum}\left\{t: C_{o}^{t} \geq T \vee C_{p}^{t} \geq T\right\}, \\
& T_{1}=\operatorname{minimum}\left\{t: C_{o}^{t} \leq T \wedge C_{p}^{t} \leq T\right\} .
\end{aligned}
$$

The graphical interpretation of these variables is shown in Fig. 6b. We set a conservative threshold value of $T=200 \mathrm{pptv}$ to remove the background noise. The choice of this time interval enables a better representation of model behaviour before and after the peak concentration.

\subsection{Puff-Movement Characteristics}

To characterize puff movements, we define several variables, which are also illustrated graphically in Fig. 6a:

- TOA-Time of arrival, which is the first time when the sensor shows a concentration reaching $10 \%$ of the maximum value.

- TOM-Time of maximum, which is the time when the sensor indicates a maximum concentration value in pptv.

- TOD-Time of departure, which is the time when the sensor, after achieving the maximum value $M V$, decreases to $10 \%$ of the value of $M V$ again.

- $M V$-Maximum value of concentration in pptv.

- DTA-Duration of time arrival, which is the difference between the time of arrival and time of maximum, DTA $=$ TOM - TOA.

- DTD-Duration of time departure, which is the difference between time of departure and time of maximum, $D T D=T O D-T O M$.

- DTR-Duration time ratio, which is the ratio between the time of arrival and departure $D T R=D T A / D T D$.

- DUR-Duration, which is the whole duration from the time of arrival to departure, $D U R=T O D-T O A=D T A+D T D$.

\section{(a)}

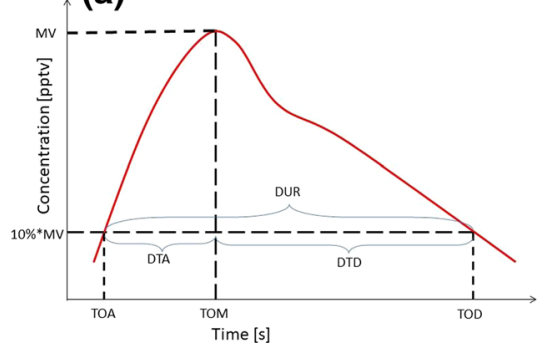

(b)

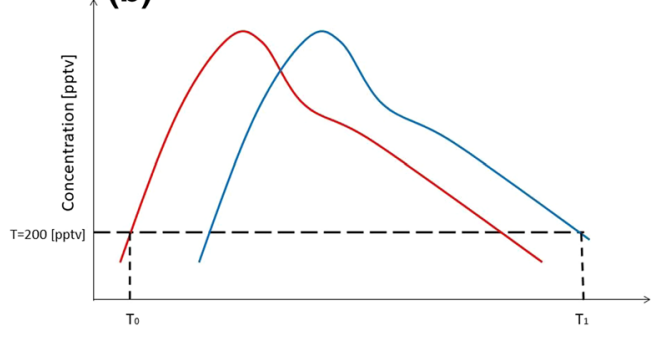

Fig. 6 a Temporal variables describing the characteristics of the puff movement. $\mathbf{b}$ The graphical interpretation of the threshold based on $T_{0}$ and $T_{1}$ values 


\section{Results}

The results of the different QUIC-model configurations are presented and compared for the instantaneous puff-release experiments during IOP 4 performed during the Joint Urban 2003 Tracer Field Experiments held in Oklahoma City from 29 June through 30 July 2003. Samplers used in this field experiment were deployed in and around the urban core of Oklahoma City, and operated by the National Oceanic and Atmospheric Administration's Air Resources Laboratory Field Research Division. One of our main goals is to validate the simulations obtained from different QUIC models with different meteorological data inputs, which is quite complex because of the need to use high-performance computing and perform multiple calculations for some cases to obtain reliable results. The QUIC program developed by the Los Alamos National Laboratory is a tool for the rapid calculation of dispersal in urban areas. For example, the QUIC-URB model is capable of calculating the pollutant dispersion about seven times faster than commonly used CFD models, and so it can be used to simulate more complex cases within the same amount of time.

For the cases considered herein, since it is clear that increasing the number of particles improves the resolution, but increases the calculation time, we use $5 \times 10^{6}$ particles, which was the maximum reasonable number for the computational power at our disposal, including parallel computing with 16 threads. The calculations were performed with the various settings presented in Sect. 3.6. The urban flow field calculated using the meteorological input data from the PWIDS-15 anemometer and from the WRF model output was determined as described in Sect. 3.2 with the QUIC-URB flow solver based on Röckle (1990). In the Q3 and Q4 cases, the flow pattern was calculated with the QUIC-CFD approach using input meteorological data from the PWIDS-15 anemometer.

The most general results regarding the simulation of gas for all models are presented in Figs. 7 and 8, showing the dosage of the $\mathrm{SF}_{6}$ gas (on a logarithmic scale) for all the model setups obtained during the three IOP 4 puffs. These values are averaged over the heights from 0 $20 \mathrm{~m}$ above ground. Each of the presented models shows a different puff trajectory; however, there are some similarities. Figure 7 show that, except for the background noise of small concentrations created by the WRF model wind profile (Q2), the plume direction and results are similar for models using the modelled WRF and observed PWIDS-15 meteorological data. The difference in the results is due to differences in meteorological stations in both cases, and the wind speed, as shown in Fig. 5. The PWIDS-15 wind-speed sensor is located on the roof of the post office, and, in the WRF model, the whole domain (shown in blue in Fig. 4) was divided into sectors with twelve grid model nodes used in the QUIC-PLUME model as sensors. Additionally, Fig. 8 shows that both puff trajectories obtained from the CFD models (cases Q3, Q4) are almost identical. The shape and direction of the plume dispersion are identical in both the CFD model set-ups, differing only in plume width because of the near-building turbulence model, which is evident in the analysis of point concentration values at the locations of the individual sensors.

\subsection{Puff 1}

Figure 9 shows each model giving varied results in comparison with the L01 sensor data. The analyzed indices for puff-movement characteristics obtained for all the IOP 4 puff 1 sensors are summarized at the top of Table 2. The two non-CFD models Q1 and Q2 show quite good results in predicting the concentrations and maximum peak. However, the models Q3 and Q4 give worse results due to the overestimation of the maximum concentration values $M V$. 

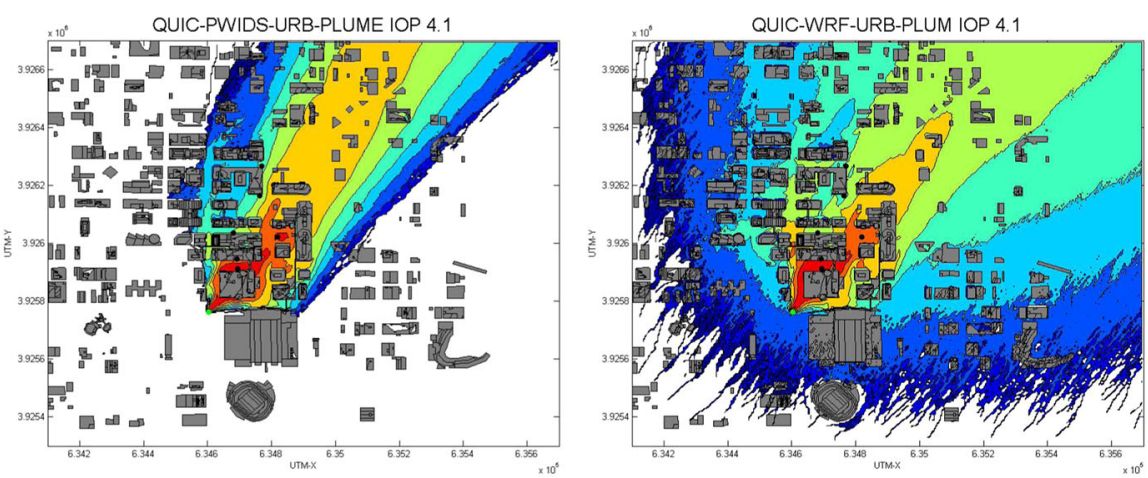

QUIC-PWIDS-URB-PLUM IOP 4.2
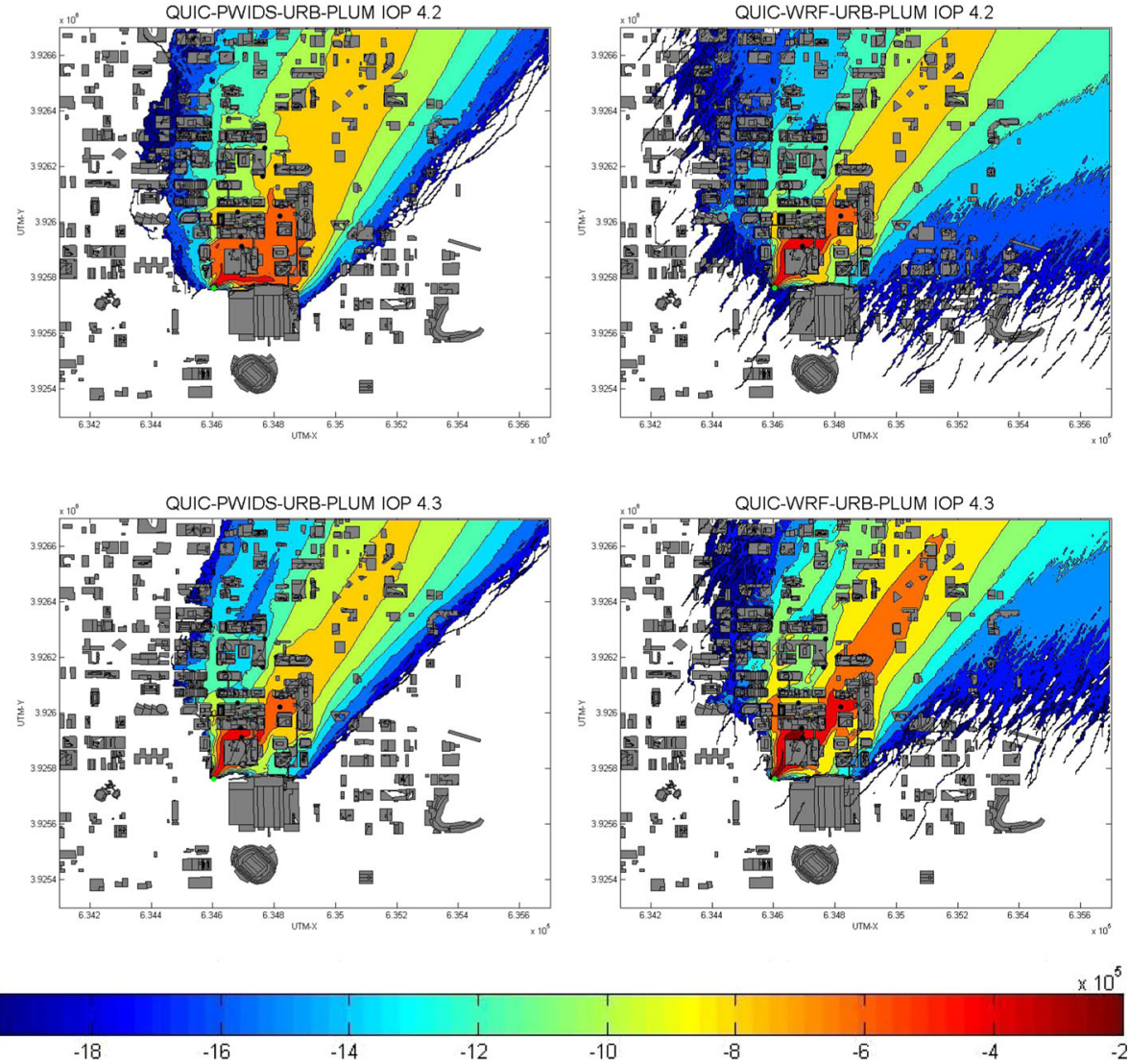

Fig. 7 Near-surface airborne mean dosage in $\ln$ (Dosage) $\left[\mathrm{kg} \mathrm{s} \mathrm{m}^{-3}\right]$ from IOP 4 using the PWIDS-URBPLUME and WRF-URB-PLUME configurations. The three rows indicate the separate puff releases of IOP 4.1, 4.2 and 4.3. The puff source was located at the Botanical Garden location shown in Fig. 1, and is indicated by the green dot. Black symbols indicate the near-surface sensor locations and one rooftop (L15) sensor location 

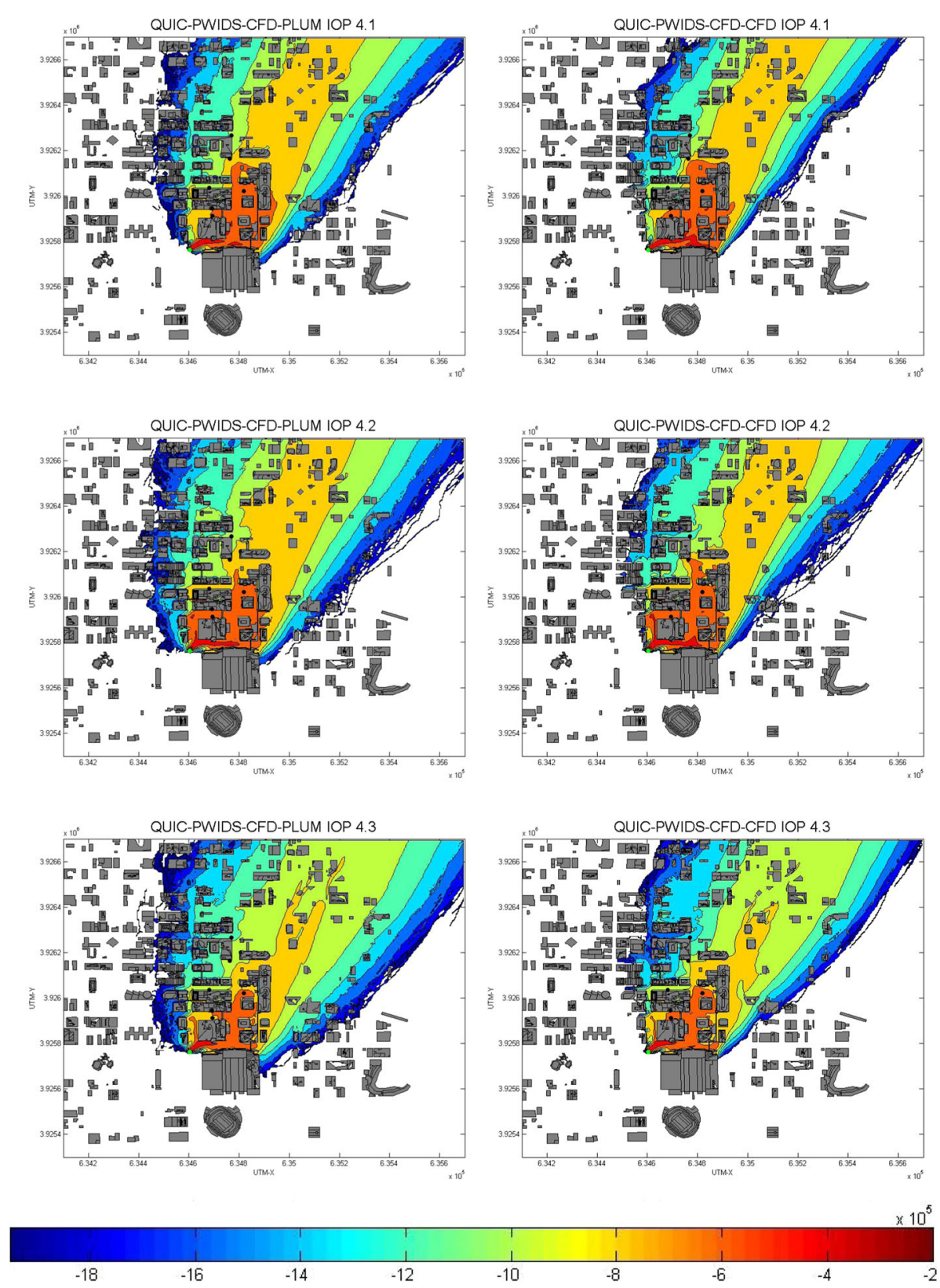

Fig. 8 Near-surface airborne mean dosage in $\ln$ (dosage) $\left[\mathrm{kg} \mathrm{s} \mathrm{m}^{-3}\right]$ from IOP 4 using the PWIDS-CFDPLUME and PWIDS-CFD-CFD configurations. The three rows indicate the separate puff releases of IOP 4.1, 4.2 and 4.3. The puff source was located at the Botanical Garden location shown in Fig. 1, and is indicated by the green dot. Black symbols indicate the near-surface sensor locations and one rooftop (L15) sensor location 

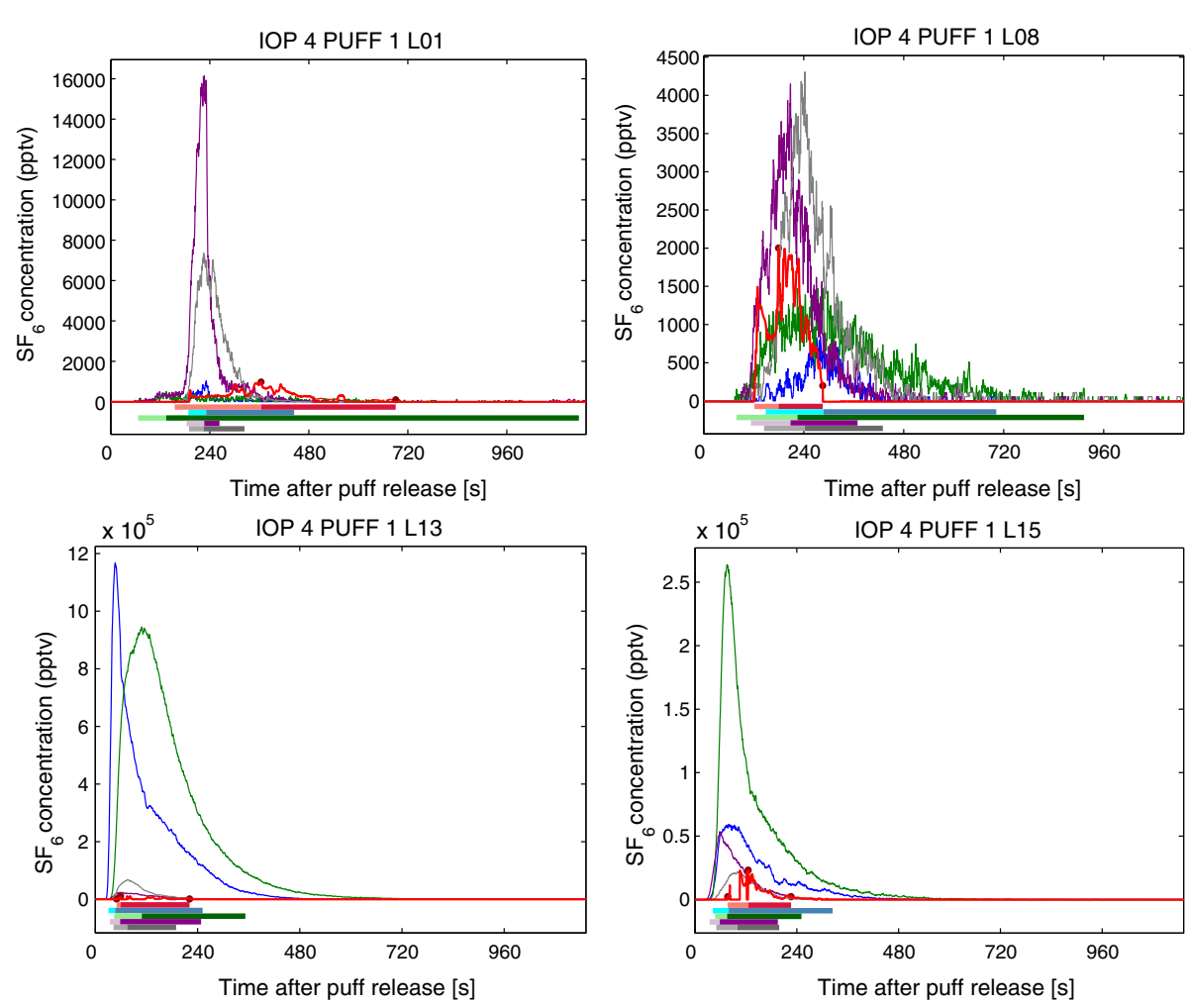

Fig. 9 Observed and modelled $\mathrm{SF}_{6}$ concentration [in pptv] time series from samplers L01, L08, L13 and L15 during puff 1 of IOP 4. Shown are the observed $\mathrm{SF}_{6}$ concentration (red), the Q1 model (blue), the Q2 model (green), the Q3 model (purple), and the Q4 model (grey) simulated values of $\mathrm{SF}_{6}$. The coloured bars indicate the intervals between the time of arrival (TOA), time of maximum value (TOM), and the time of departure $(T O D)$. Brighter shades of the same colour show intervals between the times TOA and TOM, and darker shades indicate the intervals between the times TOM and TOD

The value of the parameter DTA for sensor L01 is also underpredicted; model Q1 shows the best results in overall duration $D U R$. In the case of sensor L08, the concentration values and $M V$ parameter are similar to sensor L01, but the time parameters are slightly better predicted by the Q4 model. The value of the duration parameter for sensor L08 is somewhat larger for the models Q1 and Q2 than for the measured $S F_{6}$ data. Figure 9 shows good results for the L13 and L15 CFD models (Q3, Q4), but an overprediction by the Q1 and Q2 models, which may be caused by the location of sensors L13 and L15 (close to the puff source), while for the CFD models, the plume is nearer to the south side of the Century Center building. For the URB models, the plume is nearer to the north side of this building, which is confirmed by the trajectory of the gas cloud presented in Figs. 7-8.

For each sampler during puff 1 , we summarize the general performance metrics at the top of Table 3. The values of FAC2 for all the samplers and models fulfil the urban criterion in the following situations: the models Q1, Q2 for sensor L01, the models Q2 and Q3 for sensor L08, and the models Q3 and Q4 for sensor L15. The FB criterion is fulfilled for the Q1 model at sensor L01, the Q2 model at sensor L08, and the Q3 and Q4 models at sensor L15. The normalized mean-square-error criterion $(N M S E<6)$ is fulfilled by the models Q1 and Q2 for sensor L01, all the models for sensor L08, the model Q3 for sensor L13, and the 
models Q1, Q3, and Q4 in the case of sensor L15. Almost all indicators for the sensor L13 are far from the values required for acceptable performance. Very high values of $F B_{F P}$ suggest a systematic overprediction for all models, which means that the value of $C_{o}$ is completely enclosed by $C_{p}$. These observations are confirmed by Fig. 9. The situation is similar for the models Q1 and Q2 in the case of the sensor L15. In contrast, the CFD models (Q3, Q4) achieve a very good performance in terms of the metrics for the sensor L15, and show almost perfect correlation. In addition, the models Q3 and Q4 are very close to meeting the condition $|F B|<0.67$ for sensor $\operatorname{L08}\left(F B^{L 15}(Q 3)=-0.69, F B^{L 15}(Q 4)=-0.68\right)$. The model URB-PLUME (Q1) achieves the best results for puff 1 for the sensor L01, whereas for the WRF-based model (Q2), the best results are achieved for sensor L08.

\subsection{Puff 2}

The results for puff 2 and sensor L01 show that, except for model Q2, the models produce very good results as demonstrated in Fig. 10 and in the middle of Table 2. Model Q2 can be seen as simple noise in the concentration results. Data from sensor L08 show that only the models Q1 and Q3 generate quite good results, with model Q2 again showing noise, which, in principle, can be neglected. The intervals between TOA and TOD in these sensors are much larger than the $D U R$ parameter from the measurements. In the case of sensors L11 and L13, the L11 concentration values for all models are overpredicted by an order of magnitude, but the duration time is quite good. For sensor L13, the models Q1 and Q3 show quite good results, but the models Q4 and Q2 overestimate the concentrations and durations. The puff-parameter statistics for IOP 4 puff 2 at the L17 sensor are not so clear because of the low observed concentration values (see Table 2 and Fig. 10).

The performance measures for puff 2 are presented in the middle of Table 3, showing that, in the case of sensor L01, three models (Q1, Q3 and Q4) fulfil all criteria. For this sensor, model Q2 does not meet the success criteria for any metric. The $F B$ success criterion for sensor L08 is not fulfilled by any model, but some of the other criteria are fulfilled: FAC2 by model Q2, and NMSE by the models Q1 and Q3. We need also to mention that model Q2 is close to meeting the NMSE requirement. In the case of sensors L11 and L13, none of the models fulfil any success criteria as demonstrated in Fig. 10, showing a huge concentration difference between the measurements and model predictions. Quantitative performance metrics for sensor L17 presented in Table 3 show that, in this case, the models Q1 and Q3 fulfil all acceptance criteria. Models Q2 and Q4 give very good results with only one criterion unfulfilled $(F B)$; however, the model Q2 produces a result close to that required, $|F B|<0.67\left(F B^{L 17}(Q 2)=0.75\right)$.

\subsection{Puff 3}

In the case of puff 3 for sensor L01, the results are again not obvious because of the low concentration values (see the bottom of Table 2). However, the value of TOM for the Q4 model is almost the same as that measured. Puff-parameter statistics for sensor L011 for the CFD models (Q3, Q4) are better than for the URB models, but still not satisfactory (in particular for the value of $M V$ ), probably because of differences in the plume path as described above.

The performance measures for puff 3 are presented at the bottom of the Table 3. For sensor L11, all performance measurements are far from the acceptable urban criteria presented in Sect. 4, which is quite similar to the case of puff 1 , sensor L13. The observed measurement data $C_{o}$ are completely enclosed by all modelled values $C_{p}$ (with very high values of $F B_{F P}$ 

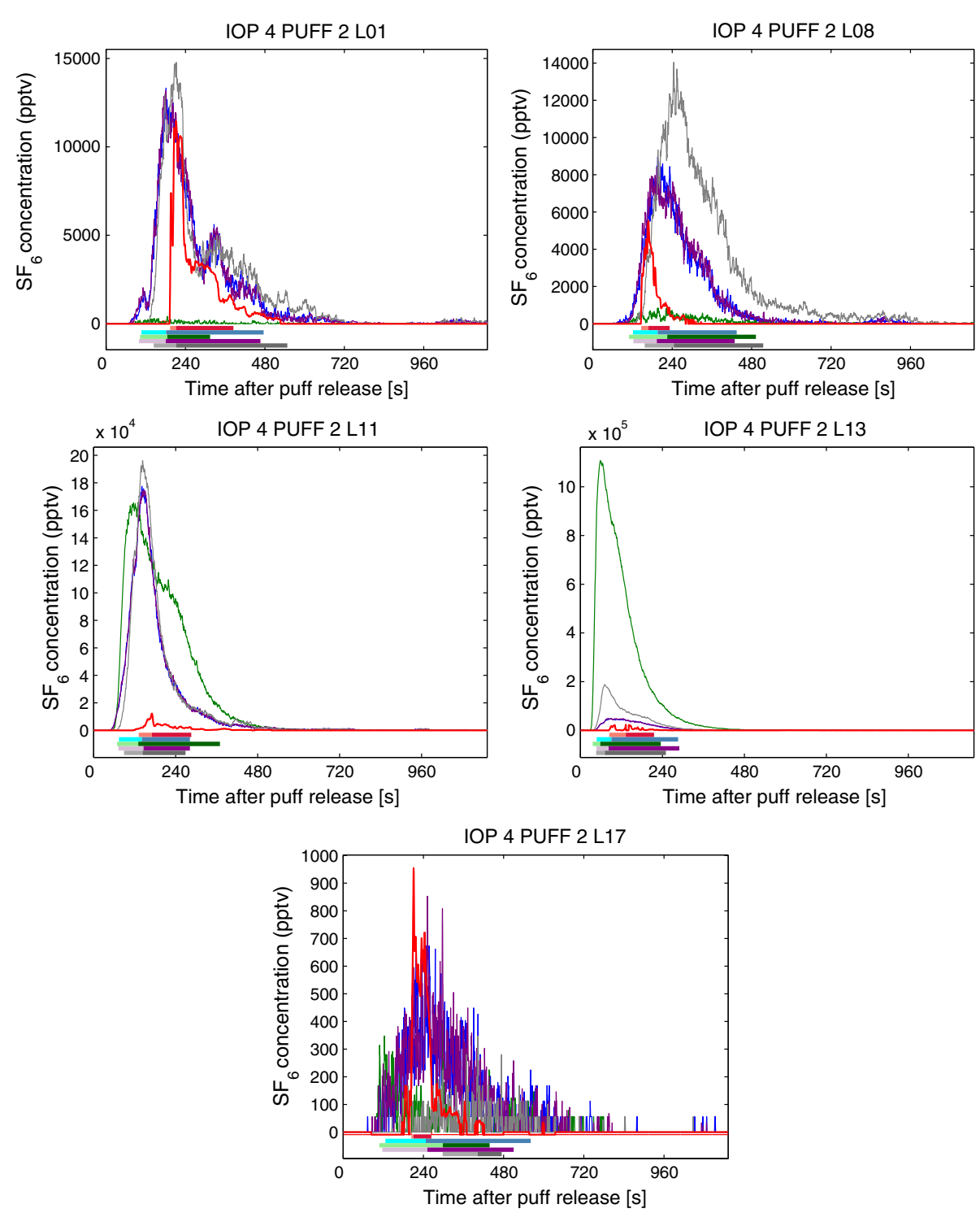

Fig. 10 Observed and modelled $\mathrm{SF}_{6}$ concentration [in pptv] time series from samplers L01, L08, L11, L13 and L17 during IOP 4 puff 2. Shown are the observed $\mathrm{SF}_{6}$ concentration (red), the model Q1 (blue), the model Q2 (green), the model Q3 (purple), and the model Q4 (grey) simulated values of $\mathrm{SF}_{6}$ concentration. The coloured bars indicate intervals between the time of arrival (TOA), time of maximum value (TOM) and time of departure (TOD). Brighter shades of the same colour show intervals between TOA and TOM, and darker shades indicate intervals between TOM and TOD 


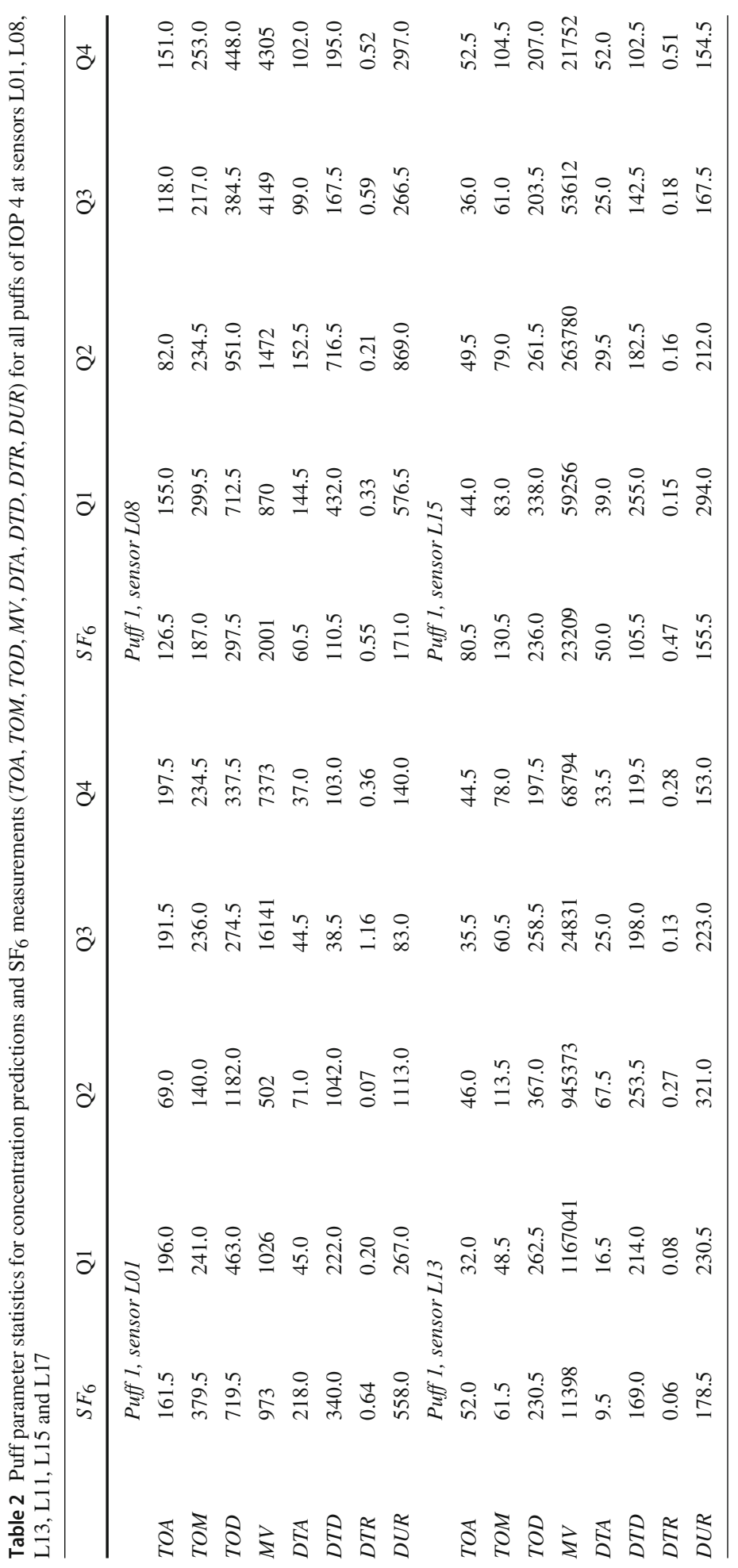




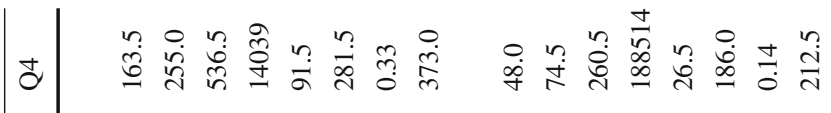

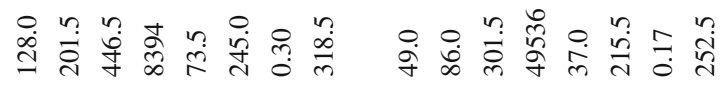

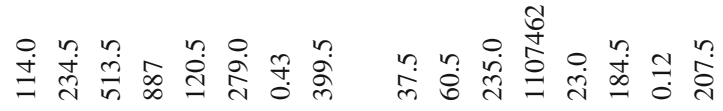

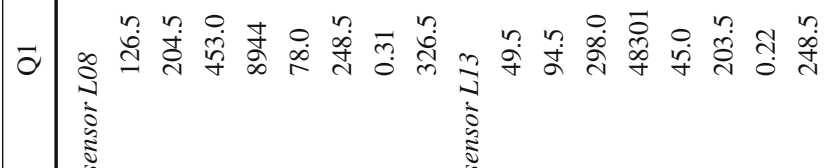

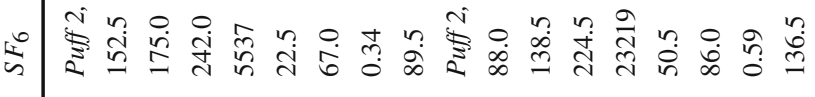

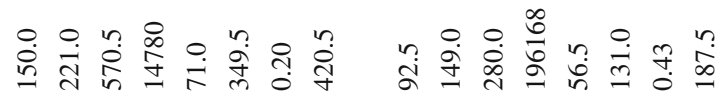

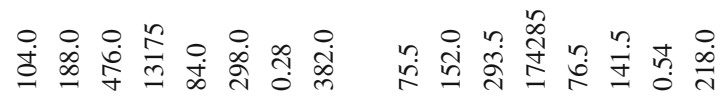

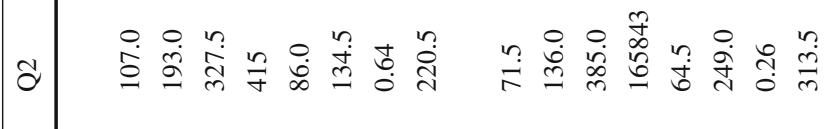

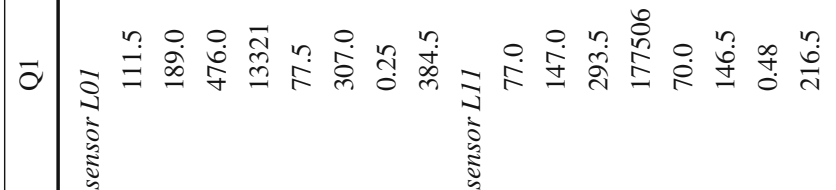

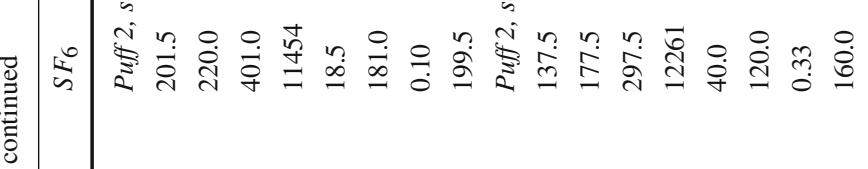

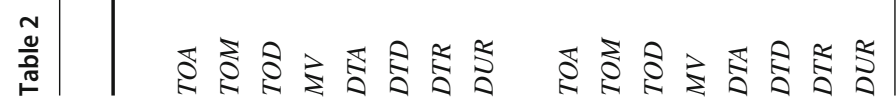




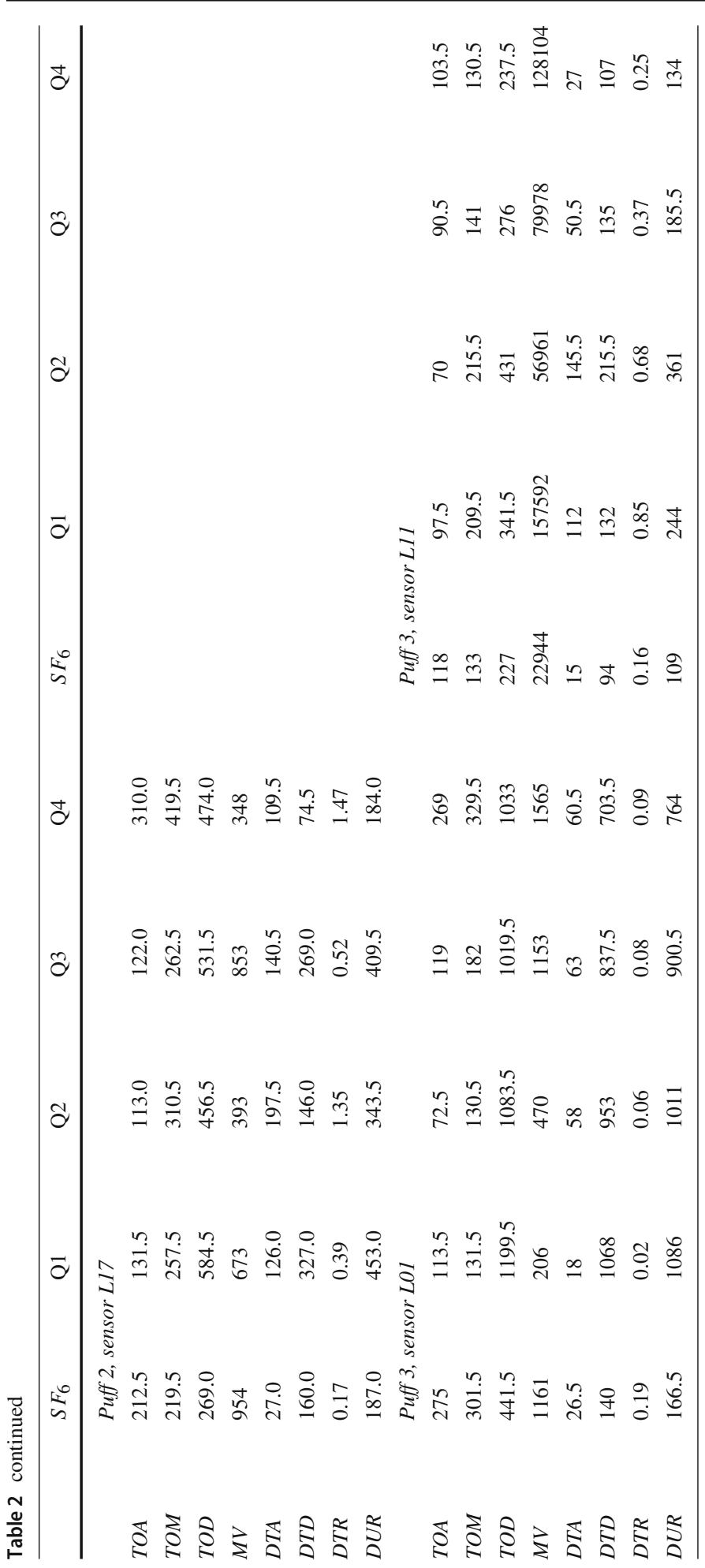


Table 3 Quantitative performance metrics for concentration predictions ( $F A C 2, F B, F B_{F N}, F B_{F P}, M G, N M S E$, $R$ ) for IOP 4 starting at 1400 UTC on 9 July 2003 for all three puffs

\begin{tabular}{|c|c|c|c|c|c|c|c|c|}
\hline \multirow{2}{*}{$\begin{array}{l}\text { Station: } \\
\text { Metric }\end{array}$} & \multicolumn{4}{|c|}{ Puff 1 , sensor L01 } & \multicolumn{4}{|c|}{ Puff 1 , sensor L08 } \\
\hline & Q1 & Q2 & Q3 & Q4 & Q1 & Q2 & Q3 & Q4 \\
\hline$F A C 2$ & 0.42 & 0.58 & 0.29 & 0.19 & 0.14 & 0.60 & 0.36 & 0.29 \\
\hline$F B$ & 0.62 & 0.75 & -1.56 & -1.44 & 0.99 & -0.06 & -0.69 & -0.68 \\
\hline$F B_{F N}$ & 0.80 & 0.76 & 0.05 & 0.08 & 1.13 & 0.29 & 0.01 & 0.12 \\
\hline$F B_{F P}$ & 0.18 & 0.01 & 1.61 & 1.51 & 0.14 & 0.34 & 0.70 & 0.79 \\
\hline$M G$ & 5.62 & 2.86 & 0.28 & 0.39 & 1.24 & 0.23 & 0.15 & 0.17 \\
\hline NMSE & 1.72 & 1.40 & 22.21 & 9.98 & 3.27 & 0.56 & 0.81 & 1.44 \\
\hline$R$ & -0.37 & -0.20 & -0.47 & -0.57 & -0.16 & 0.18 & 0.88 & 0.44 \\
\hline Station: & \multicolumn{4}{|c|}{ Puff 1, sensor L13 } & \multicolumn{4}{|c|}{ Puff 1, sensor L15 } \\
\hline Metric: & Q1 & Q2 & Q3 & Q4 & Q1 & Q2 & Q3 & Q4 \\
\hline$F A C 2$ & 0.00 & 0.00 & 0.15 & 0.07 & 0.03 & 0.00 & 0.66 & 0.54 \\
\hline$F B$ & -1.97 & -1.99 & -1.13 & -1.43 & -1.18 & -1.63 & -0.22 & 0.01 \\
\hline$F B_{F N}$ & 0.00 & 0.00 & 0.00 & 0.00 & 0.00 & 0.00 & 0.06 & 0.14 \\
\hline$F B_{F P}$ & 1.97 & 1.99 & 1.13 & 1.43 & 1.18 & 1.63 & 0.28 & 0.14 \\
\hline$M G$ & 0.00 & 0.00 & 0.05 & 0.04 & 0.16 & 0.06 & 0.95 & 1.54 \\
\hline NMSE & 186.63 & 489.10 & 4.44 & 13.07 & 2.69 & 10.28 & 0.29 & 0.17 \\
\hline$R$ & 0.61 & 0.67 & 0.61 & 0.52 & 0.92 & 0.89 & 0.91 & 0.93 \\
\hline Station: & \multicolumn{4}{|c|}{ Puff 2, sensor L01 } & \multicolumn{4}{|c|}{ Puff 2, sensor L08 } \\
\hline Metric: & Q1 & Q2 & Q3 & Q4 & Q1 & Q2 & Q3 & Q4 \\
\hline$F A C 2$ & 0.70 & 0.11 & 0.61 & 0.62 & 0.18 & 0.47 & 0.17 & 0.24 \\
\hline$F B$ & -0.56 & 1.84 & -0.58 & -0.62 & -1.24 & 1.04 & -1.25 & -1.45 \\
\hline$F B_{F N}$ & 0.02 & 1.84 & 0.02 & 0.01 & 0.00 & 1.13 & 0.00 & 0.05 \\
\hline$F B_{F P}$ & 0.59 & 0.00 & 0.61 & 0.63 & 1.24 & 0.08 & 1.25 & 1.51 \\
\hline$M G$ & 0.42 & 49.09 & 0.41 & 0.39 & 0.09 & 0.99 & 0.09 & 0.13 \\
\hline NMSE & 0.94 & 39.31 & 0.97 & 0.88 & 3.36 & 6.17 & 3.23 & 7.31 \\
\hline$R$ & 0.33 & 0.13 & 0.31 & 0.57 & 0.32 & 0.12 & 0.43 & -0.39 \\
\hline Station: & \multicolumn{4}{|c|}{ Puff 2, sensor L11 } & \multicolumn{4}{|c|}{ Puff 2, sensor L13 } \\
\hline Metric: & Q1 & Q2 & Q3 & Q4 & Q1 & Q2 & Q3 & Q4 \\
\hline$F A C 2$ & 0.00 & 0.00 & 0.00 & 0.00 & 0.03 & 0.00 & 0.02 & 0.00 \\
\hline$F B$ & -1.83 & -1.89 & -1.83 & -1.85 & -1.49 & -1.95 & -1.50 & -1.72 \\
\hline$F B_{F N}$ & 0.00 & 0.00 & 0.00 & 0.00 & 0.00 & 0.00 & 0.00 & 0.00 \\
\hline$F B_{F P}$ & 1.83 & 1.89 & 1.83 & 1.85 & 1.49 & 1.95 & 1.50 & 1.72 \\
\hline$M G$ & 0.05 & 0.02 & 0.05 & 0.05 & 0.10 & 0.01 & 0.09 & 0.05 \\
\hline
\end{tabular}


Table 3 continued

\begin{tabular}{|c|c|c|c|c|c|c|c|c|}
\hline \multirow{2}{*}{$\begin{array}{l}\text { Station: } \\
\text { Metric: }\end{array}$} & \multicolumn{4}{|c|}{ Puff 2, sensor L11 } & \multicolumn{4}{|c|}{ Puff 2, sensor L13 } \\
\hline & Q1 & Q2 & Q3 & Q4 & Q1 & Q2 & Q3 & Q4 \\
\hline NMSE & 42.96 & 42.22 & 43. & 48.42 & 7.96 & 150.78 & 8.22 & 18.93 \\
\hline$R$ & 0.53 & 0.51 & 0.5 & 0.55 & 0.56 & 0.47 & 0.56 & 0.53 \\
\hline Station: & \multicolumn{7}{|c|}{ Puff 2, sensor L17 } & \\
\hline Metric: & \multicolumn{2}{|c|}{ Q1 } & \multicolumn{2}{|c|}{ Q2 } & Q3 & \multicolumn{2}{|r|}{ Q4 } & \\
\hline$F A C 2$ & \multicolumn{2}{|c|}{0.87} & \multicolumn{2}{|c|}{0.50} & 0.78 & \multicolumn{2}{|r|}{0.45} & \\
\hline$F B$ & \multicolumn{2}{|c|}{0.00} & \multicolumn{2}{|c|}{0.75} & -0.06 & \multicolumn{3}{|c|}{0.94} \\
\hline$F B_{F N}$ & \multicolumn{2}{|c|}{0.19} & \multicolumn{2}{|c|}{0.76} & 0.19 & \multicolumn{3}{|c|}{0.94} \\
\hline$F B_{F P}$ & \multicolumn{2}{|c|}{0.19} & \multicolumn{2}{|c|}{0.01} & 0.25 & \multicolumn{3}{|c|}{0.00} \\
\hline$M G$ & \multicolumn{2}{|c|}{0.92} & \multicolumn{2}{|c|}{6.22} & 0.89 & \multicolumn{3}{|c|}{13.22} \\
\hline NMSE & \multicolumn{2}{|c|}{0.30} & \multicolumn{2}{|c|}{1.59} & 0.35 & \multicolumn{3}{|c|}{2.55} \\
\hline$R$ & \multicolumn{2}{|c|}{0.39} & \multicolumn{2}{|c|}{0.03} & 0.31 & \multicolumn{3}{|c|}{-0.19} \\
\hline Station: & \multicolumn{4}{|c|}{ Puff 3, sensor L01 } & \multicolumn{4}{|c|}{ Puff 3, sensor L11 } \\
\hline Metric: & Q1 & Q2 & Q3 & Q4 & Q1 & Q2 & Q3 & Q4 \\
\hline$F A C 2$ & 0.11 & 0.28 & 0.77 & 0.74 & 0.02 & 0.00 & 0.02 & 0.01 \\
\hline$F B$ & 1.34 & 0.88 & -0.08 & -0.43 & -1.75 & -1.56 & -1.32 & -1.45 \\
\hline$F B_{F N}$ & 1.38 & 0.92 & 0.16 & 0.04 & 0.00 & 0.00 & 0.00 & 0.00 \\
\hline$F B_{F P}$ & 0.03 & 0.04 & 0.24 & 0.47 & 1.75 & 1.56 & 1.32 & 1.45 \\
\hline$M G$ & 23.98 & 1.98 & 0.70 & 0.71 & 0.04 & 0.05 & 0.13 & 0.11 \\
\hline NMSE & 5.09 & 1.76 & 0.27 & 0.45 & 19.26 & 6.97 & 5.76 & 9.37 \\
\hline$R$ & 0.01 & 0.05 & 0.41 & 0.68 & 0.20 & 0.47 & 0.78 & 0.82 \\
\hline
\end{tabular}

Measurements are from sensors L01, L08, L13, L11, L15 and L17
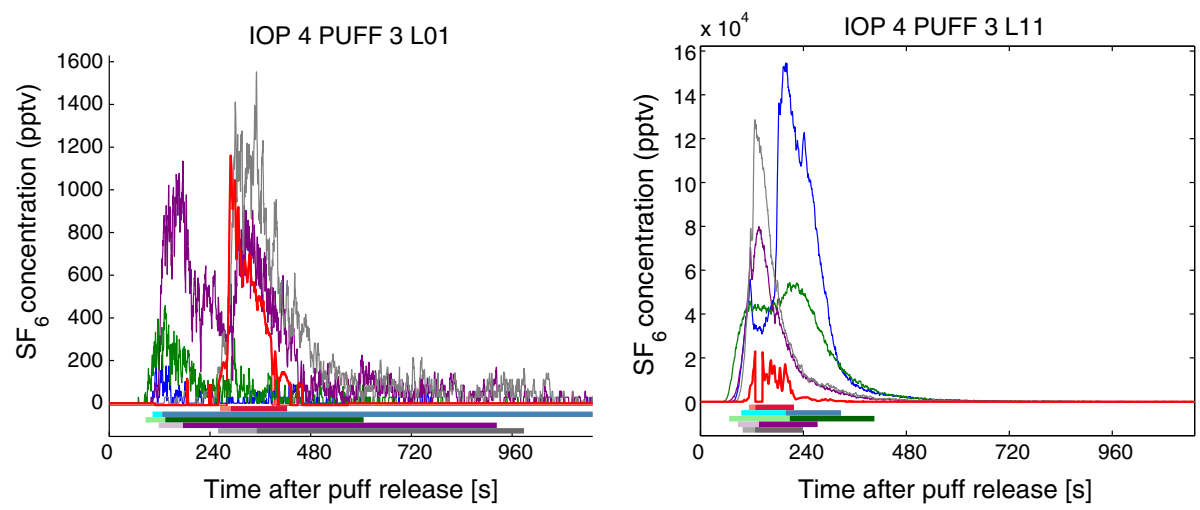

Fig. 11 Observed and modelled $\mathrm{SF}_{6}$ concentration (in pptv) time series from samplers L01 and L11 during puff 3 of IOP 4. Shown are the observed $\mathrm{SF}_{6}$ concentration (red), the Q1 model (blue), the Q2 model (green), the Q3 model (purple), and the Q4 model (grey) for the simulated values of $\mathrm{SF}_{6}$. The coloured bars indicate the intervals between the time of arrival (TOA), time of maximum value (TOM) and time of departure (TOD). Brighter shades of the same colour show intervals between TOA and TOM, and darker shades indicate intervals between TOM and TOD 
for all models), which is also confirmed by Fig. 11. In contrast, all the criteria are fulfilled by models Q3 and Q4, when taking into account the sensor L01. The NMSE criterion for sensor L01 is fulfilled for all QUIC model set-ups.

\section{Conclusions}

We have demonstrated the good performance of the QUIC-URB and QUIC-CFD models for the same cases, with differences mainly caused by the different flow models. In some cases, as can be seen from Figs. 7 and 8, the flow near the Oklahoma Century Center building diverges in different directions. For the QUIC-URB models, in most cases, the plume is transported to the north side of the Century Center building (except for the QUIC-PWIDS-URB-PLUME model for IOP 4.2), resulting in higher concentrations for sensors L13 and L15. In contrast, lower concentrations are predicted at these sensor locations by the CFD-type models due to the plume moving mostly to the south side of the Century Center building. Additionally, we need to mention that the plume paths for the PWIDS-URB and PWIDS-CFD models for IOP 4.2 are similar. Finally, these differences lead to various values of the indicators used for performance measures, and there is no clear advantage of one model over the others. In the considered cases, each of the models produced both a worse and better performance depending on the metric considered. Therefore, in order to better understand the reasons for the model behaviour, further validation is needed (for example, sensitivity analyses), with this work serving as a reference for further research. In particular, it would be convenient to have a sufficiently dense network of sensors to compare the observed and predicted concentrations across a wider spatial area. It should be also mentioned that, in general, the QUIC program is able to predict reasonable results quite rapidly for complex urban areas and, therefore, can be applied in real emergency preparedness and response events.

Acknowledgements We gratefully acknowledge the European Commission Directorate General for Migration and Home Affairs (DG HOME) for their support of the UDINEE activity. The authors want to acknowledge the contribution of various groups to the UDINEE project. The following agencies have prepared the datasets used here: U.S. Army Dugway Proving Group as manager of the JU2003 database; data from tracer monitoring stations were provided by the National Oceanic and Atmospheric Administration Air Resources Laboratory Field Research Division; data from meteorological monitoring stations were provided by Dugway Proving Ground. The Joint Research Center Ispra/Institute for Environment and Sustainability provided its ENSEMBLE system for model output harmonization and analyses and evaluation.

Open Access This article is distributed under the terms of the Creative Commons Attribution 4.0 International License (http://creativecommons.org/licenses/by/4.0/), which permits unrestricted use, distribution, and reproduction in any medium, provided you give appropriate credit to the original author(s) and the source, provide a link to the Creative Commons license, and indicate if changes were made.

\section{References}

Brown MJ, Gowardhan AA, Nelson MA, Williams MD, Pardyjak ER (2013) QUIC transport and dispersion modelling of two releases from the Joint Urban 2003 field experiment. Int J Environ Pollut 52(3-4):263287

Chang JC, Hanna SR (2005) Technical descriptions and user's guide for the BOOT statistical model evaluation software package, version 2.0. George Mason University and Harvard School of Public Health, Fairfax, Virginia, USA

Clawson KL, Carter RG, Lacroix DJ, Biltoft CJ, Hukari NF, Johnson RC, Rich JD, Beard SA, Strong T (2005) Joint Urban 2003 (JU2003) SF6 Atmospheric Tracer Field Tests. Air Resources Laboratory, Silver Spring, Maryland, USA. NOAA Tech Memo OAR ARL-254 
Chorin AJ (1968) Numerical solution of the Navier-Stokes equations. Math Comput 22(104):745-762

Gowardhan AA, Pardyjak ER, Senocak I, Brown MJ (2011) A CFD-based wind solver for an urban fast response transport and dispersion model. Environ Fluid Mech 11(5):439-464

Hanna S, Chang J (2012) Acceptance criteria for urban dispersion model evaluation. Meteorol Atmos Phys 116(3-4):133-146

Hanna SR, Brown MJ, Camelli FE, Chan ST, Coirier WJ, Kim S, Hansen OR, Huber AH, Reynolds RM (2006) Detailed simulations of atmospheric flow and dispersion in downtown Manhattan: an application of five computational fluid dynamics models. Bull Am Meteorol Soc 87(12):1713-1726

Hernández-Ceballos MA, Hanna S, Bianconi R, Bellasio R, Mazzola T, Chang J, Andronopoulos S, Armand P, Benbouta N, Carný P, Ek N, Fojcí-ková E, Fry R, Huggett L, Kopka P, Korycki M, Lipták L, Millington S, Miner S, Oldrini O, Potempski S, Tinarelli G, Trini Castelli S, Venetsanos A, Galmarini S (2017) UDINEE: evaluation of multiple models with data from the JU2003 puff releases in Oklahoma City. Part I: comparison of observed and predicted concentrations. Boundary-Layer Meteorol (submitted for publication)

Nelson MA, Brown MJ, Halverson SA, Bieringer PE, Annunzio A, Bieberbach G, Meech S (2016) A case study of the weather research and forecasting model applied to the Joint Urban 2003 tracer field experiment. Part 2: gas tracer dispersion. Bound Layer Meteorol 161(3):461-490

Pardyjak ER, Brown M (2003) QUIC-URB v. 1.1: Theory and User's Guide. Los Alamos National Laboratory, Los Alamos, New Mexico, USA

Röckle R (1990) Bestimmung der stomungsverhaltnisse im bereich komplexer bebauungsstrukturen. PhD thesis, Vom Fachbereich Machanik, der Technischen Hochshule, Darmstadt, Germany (in German)

Sherman CA (1978) A mass-consistent model for wind fields over complex terrain. J Appl Meteorol 17(3):312319

Williams MD, Brown MJ, Singh B, Boswell D (2004) QUIC-PLUME theory guide. Los Alamos National Laboratory, $43 \mathrm{pp}$

Wilson JD, Yee E, Ek N, d'Amours R (2009) Lagrangian simulation of wind transport in the urban environment. Q J R Meteorol Soc 135:1586-1602

Wyszogrodzki AA, Miao S, Chen F (2012) Evaluation of the coupling between mesoscale-WRF and LESEULAG models for simulating fine-scale urban dispersion. Atmos Res 118:324-345 